\title{
Corrigendum: Isoform Age - Splice Isoform Profiling Using Long-Read Technologies
}

\author{
Ricardo De Paoli-Iseppi ${ }^{\dagger}$, Josie Gleeson ${ }^{\dagger}$ and Michael B. Clark* \\ Centre for Stem Cell Systems, Department of Anatomy and Physiology, The University of Melbourne, Parkville, VIC, Australia
}

Keywords: isoform, long-read sequencing, PacBio, Oxford Nanopore Technologies nanopore sequencing, single cell sequencing, alternative splicing, spatial transcriptomics, targeted RNA sequencing

\section{Corrigendum on}

Isoform Age - Splice Isoform Profiling Using Long-Read Technologies

by De Paoli-Iseppi, R., Gleeson, J., and Clark, M. B. (2021). Front. Mol. Biosci. 8, 744. doi: 10.3389/ fmolb.2021.711733

In the original article, the reference for Kahraman et al. (2020) was incorrectly written as "Kahraman, A., Karakulak, T., Szklarczyk, D., von Mering, C., Kahraman, A., Karakulak, T., et al. (2020). Pathogenic Impact of Transcript Isoform Switching in 1,209 Cancer Samples Covering 27 Cancer Types Using an Isoform-specific Interaction Network. Sci. Rep. 10, 14453. doi:10.1038/s41598-020-71221-5.”

The correct reference is "Kahraman, A., Karakulak, T., Szklarczyk, D., and von Mering, C. (2020).

\section{OPEN ACCESS}

Edited and reviewed by: Abdullah Kahraman, University Hospital Zürich, Switzerland

${ }^{*}$ Correspondence: Michael B. Clark michael.clark@unimelb.edu.au

${ }^{\dagger}$ These authors have contributed equally to this work

Specialty section:

This article was submitted to Protein and RNA Networks,

a section of the journal

Frontiers in Molecular Biosciences

Received: 31 August 2021 Accepted: 13 September 2021 Published: 27 September 2021

Citation:

De Paoli-Iseppi R, Gleeson J and Clark MB (2021) Corrigendum: Isoform Age - Splice Isoform Profiling Using Long-Read Technologies.

Front. Mol. Biosci. 8:767743.

doi: 10.3389/fmolb.2021.767743
Pathogenic Impact of Transcript Isoform Switching in 1,209 Cancer Samples Covering 27 Cancer Types Using an Isoform-specific Interaction Network. Sci. Rep. 10, 14453. doi:10.1038/s41598-02071221-5."

Additionally, there was an error in the Discussion section, which meant one sentence did not convey the intended meaning. A correction has been made to Discussion, Paragraph 1:

"Long-read sequencing enables profiling of full-length RNA and cDNA reads, which is essential for mapping alternative RNA isoforms in tissues and disease states. Coupling longread data with both short reads and cutting-edge technologies such as single cell sequencing significantly widens the toolset for accurate isoform discovery in complex transcriptomes. Long read methods currently involve a trade-off between higher accuracy (Hifi, R2C2) and higher throughput (Nanopore, PacBio subreads). While lower accuracy can necessitate sophisticated error correction tools and/or paired short-read data, the advantages of longover short-reads for isoform detection and quantification already outweigh many of the drawbacks in error rate (Chen et al., 2021). Furthermore, we anticipate that error correction will not be necessary in future due to the rapid pace at which long-read technologies are improving."

The authors apologize for these errors and state that they do not change the scientific conclusions of the article in any way. The original article has been updated.

\section{REFERENCES}

Chen, Y., Davidson, N. M., Wan, Y. K., Patel, H., Yao, F., Low, H. M., et al. (2021). A Systematic Benchmark of Nanopore Long Read RNA Sequencing for Transcript Level Analysis in Human Cell Lines. bioRxiv, 440736. doi:10.1101/ 2021.04.21.440736 
Kahraman, A., Karakulak, T., Szklarczyk, D., and von Mering, C. (2020). Pathogenic Impact of Transcript Isoform Switching in 1,209 Cancer Samples Covering 27 Cancer Types Using an Isoform-specific Interaction Network. Sci. Rep. 10, 14453. doi:10.1038/s41598-020$71221-5$

Publisher's Note: All claims expressed in this article are solely those of the authors and do not necessarily represent those of their affiliated organizations, or those of the publisher, the editors and the reviewers. Any product that may be evaluated in this article, or claim that may be made by its manufacturer, is not guaranteed or endorsed by the publisher.

Copyright $\odot 2021$ De Paoli-Iseppi, Gleeson and Clark. This is an open-access article distributed under the terms of the Creative Commons Attribution License (CC BY). The use, distribution or reproduction in other forums is permitted, provided the original author(s) and the copyright owner(s) are credited and that the original publication in this journal is cited, in accordance with accepted academic practice. No use, distribution or reproduction is permitted which does not comply with these terms. 